\title{
What Nanobacteria and Nanovesicles May Tell Us about the Origin of Life?
}

\author{
Igor Jerman \\ General Department, Institute Bion, Ljubljana, Slovenia \\ Email: igor.jerman@bion.si
}

How to cite this paper: Jerman, I. (2017) What Nanobacteria and Nanovesicles May Tell Us about the Origin of Life? Open Access Library Journal, 4: e3348.

http://dx.doi.org/10.4236/oalib.1103348

Received: December 31, 2016

Accepted: January 20, 2017

Published: January 23, 2017

Copyright $\odot 2017$ by author and Open Access Library Inc.

This work is licensed under the Creative Commons Attribution International License (CC BY 4.0).

http://creativecommons.org/licenses/by/4.0/

\section{(c) (i) Open Access}

\begin{abstract}
In contemporary, established biology life is almost exclusively treated as a molecular phenomenon. Therefore, the mystery of the origin of life is sought in molecular terms and processes. But according to certain advanced researches and considerations, life has also other essential "ingredients": active and diffused organized information and a specific physical as well as physicochemical state of matter characterized by long range order and coherent domains. These characteristics should also form the basis of the pre-biotic evolution, the phase of more or less organized nano- and micro-vesicular systems that lead from abiotic to living systems. In this view, the complex molecular, physical and physicochemical order replaces the DNA molecule in its capability to maintain the stability of information complexity from generation to generation. Such systems were already found on the present day Earth and even within organisms and were also synthetically reproduced. They are called nanobacteria and in general nanovesicles or nanoparticles. They may represent an actual passage from non living forms to primitive organisms.
\end{abstract}

\section{Subject Areas \\ Evolutionary Studies}

\section{Keywords}

Origin of Life, Definition of Life, Pre-Biotic Evolution, Coherent Domains, Long Range Order, Active Information, Nanobacteria, Bions, Biomimetic, Biomorphs

\section{Introduction}

\section{Molecular View of Life and Its Origin}

Until Pasterur's famous experiment that yielded the proverb "Omne vivum ex vivo" (all living from the living), the origin of life was not regarded as a problem. 
Biological thinkers believed in the so called generatio spontanea, i.e. spontaneous generation of life from the inanimate matter at least at the level of microorganisms. Pasteur sealed that view and opened an as yet insurmountable question, namely, how then life emerged in the first place, whether on the Earth or elsewhere in cosmos (so called Panspermian hypotheses). After Pasteur's experiment it became obvious that contemporary inanimate nature has no power to spontaneously generate life, yet it was also (and still is) logical that there was time in the past of the Earth when there was no life at all. Therefore, by necessity there had to be time that life emerged from the inanimate nature, i.e. from the non living nature. Through many years of trying to solve this new enigma three main ideas appeared:

1) The emergence of life on the Earth was just a highly improbable event, never to be repeated again. It is only a high local aberration, nothing at home in the Universe. There are no laws leading nonliving matter to life, it was just a pure luck that happened just on the Earth soon after its formation.

2) The emergence of life was due to a) a special living force or b) of God's creation. In both cases, it would emerge from causes extraneous to matter in which it emerged and has evolved.

3) The emergence was due to laws of self-organization of chemical processes under certain circumstances. Life's origin is therefore a law-like process (event) and could be widespread in cosmos and even in our galaxy. As any lawful physical event the emergence of life would need to have fulfilled certain conditions, after which it would appear with a high probability if not with certainty. The role of a biological (biochemical) scientist would then only consist in identifying these laws and consequently trying to reproduce the origin of life in the laboratory where suitable substances would be subject to proper conditions.

As the first two views leave no room for scientific experimentation and equally so for their rejection, they may be safely regarded as unscientific. The third one, however, is scientific in nature, since it enables a scientist to make hypotheses and test them. But in spite of many theoretical considerations and countless experiments probing the assumed primordial Earth conditions we are still in darkness as to any true and satisfying mechanism explaining the path from simple chemicals to the simplest living system. Mostly, the research efforts are concentrated on self-organizing capabilities of autocatalytic chemical systems to have a sort of evolution, i.e. a stable line from a simple to more complex formations.

In all these approaches, life as well as its origin are regarded basically and almost totally as a molecular phenomenon within certain physical limits (the laws of entropy for instance). Besides pure chemistry, the self-organization approach uses also more subtle entities like information, complexity, order etc., but it binds all these attributes again to purely chemical processes. Even one of the most modern physically based hypotheses proposed by England [1] - arguing that the origin of life is due to the laws of entropy and that it is consequently a 
highly probable event-is based on a conviction that life is in the first place a complex molecular phenomenon. However, the exclusively chemical outlook on life may not be a fruitful attitude; it may be just a cul-de-sac that will never explain either life itself or its origin. Life may just simply be a much more complex and robust phenomenon from what is visualized by the majority of contemporary biologists. From its very beginning it may be hierarchically organized; it may rest not only on molecules and their laws, but also on certain physical properties of colloidal systems, interfacial water and endogenous electromagnetic fields, as it was elaborated in my previous publications [2] [3]. As it was disclosed there, this larger issue in treating life opens also a fresh horizon for the solution of the origin of life problem. In the following I will present a challenge that contemporary life may be surrounded by more primitive formations, various physicochemical ${ }^{1}$ vesicles that may form a living bridge between unquestionably living forms and non living matter. We will speak about nanobacteria, bions and some other similar vesicular formations and see what they can tell us about the origin of life.

\section{Views of Life and Water That Promises Fresh Possibilities to Disentangle the Mystery of the Origin of Life}

\subsection{Life as Organized Information}

Contemporary biology is permeated with informational terminology connected to the envisioned chemical nature of life. Organisms are consequently seen as very complex information processing molecular systems. Basically, if we focus ourselves on purely chemical level, we have two types of molecular information:

a) The passive one, storing organism's complexity (genetic information, seen as residing mostly in genes, "DNA") and

b) The active, manipulative one, mostly working through proteins.

Since even with the simplest organisms all information needed to process life is hierarchically organized, even in an unicellular organism that may be seen as the smallest self-sustaining living entity, it should be processed at least on two levels:

a) On the already mentioned biochemical level and

b) On the cellular level. Here the organized information should connect complex biochemistry with various fields that were discussed at length in my previous article [3] and will be here shortly presented later.

Of course, there are higher levels of information processing (dynamic organization) that would be recognized as physiological, behavioral or psychological (bound mostly to a complex nervous system in animals and humans), but they do not concern the problem of the origin of life. Even in the simplest organisms this organized information (2 types-static, dynamic, and 2 above introduced levels) should have also a capacity for self propagation through similar systems (information carriers and processors: organisms).

According to a recent consideration [4] the starting point for the emergence

${ }^{1}$ i.e. Obeying not only chemical but also physical laws. 
of life should mean an establishment of causal powers from the side of organized information over matter, where the former is encrypted in a disperse way. In other words, the causal power of life is trans-material, whereby we do not introduce vis vitalis or some other mystic force, but the dynamically organized information. Only partially this dispersed and organized information "resides" in the DNA. The organisms are seen here as the so called non-trivial duplicators, systems that can:

a) Multiply their organization (i.e. organized information) in a complex and widely dispersed way and

b) Simultaneously actively process information and express a high degree of flexibility (variability).

According to this advanced view, the origin of life means an establishment of organized information, capable of further potentially limitless enrichment of its content (complexity) and its dynamic organization. With the origin of life information as an active principle of nature gains the ability of causation from the top to bottom (from the whole to its parts, from the cell to its molecules). It ensures holism of living cells. Here we are faced with a relatively new notion of information, sharing some basic connotations with the Shannon's information theory, yet at the same time far exceeding it. As an active principle behind living processes, information arises as a powerful natural principle. It works in and through mater, yet it is casually "above" it. Life, therefore, has its special principle that surpasses ordinary (inanimate) physical and chemical world, but is on the other hand a natural principle, like matter, time, space etc. This principle resides in the inanimate matter as a potential-and emerges when the suitable conditions arise. In the following we shall try to elaborate a little more these possible conditions and propose a hypothesis that they are not so exceptional and bound only to some distant past on the Earth or even some distant part of the universe.

\subsection{Biophysical Basis of Life}

Since the reductionist biology has began to establish itself (it started during the second half of the $19^{\text {th }}$ century), the minority of biologists opposed to the more and more "molecularized" outlook on life. They sought a unifying principle that could explain the immense orderliness and complexity found even in the simplest organisms. They were accused of vitalism from the established majority and at least with the start of the DNA era in biology certain lines of vitalistic thought were really banned from biology. However, the spark of a holistic, emergentist, organistic outlook on life was never extinguished; during the $20^{\text {th }}$ century it simply moved from a prevailing mystical perception to a more scientific one.

Some biologists realized that at least during embryogenesis the developmental processes can be understood and explained through the terminology of fields whose nature may be unknown, but that hold some similarity to physical fields [5] [6]. The biggest difference was that the "biological" (morphogenetic) fields 
were very dynamic - they have a very complex chreod (attractor path) from the zygote to the fully grown individual, pierced by many points of symmetry breaking.

On the other hand and stemming from a purely physical reasoning in the sixties of the past century, Herbert Fröhlich found that the cellular state rests on rhythmic and coherent oscillations of polar (macro)molecules (called also polarons, see [7]) and that these oscillations can bring much order even into cellular biochemistry [8] [9]. Fröhlich's ideas were later elaborated by an Italian group of quantum physicists (Del Giudice, Vitiello, Preparata) on the level of quantum field theory. The theory could be called the theory of quantum bio-electrodynamics [3] [10] [11] [12]. From the theory it stems that life at the cellular level (biochemistry, molecular movements) is highly organized through a filamentous coherent (i.e. laser like) electromagnetic field. Further considerations on this line are given at length by Meijer and Geesink [7].

The quest for a more comprehensive and unifying factor of life therefore found a very fruitful foundation in a special quantum field that underlines organism's life and is seen as at least partially organizing its biochemistry. Consequently the latter is not subject only to randomness of Brownian molecular movements, but also to an organizing principle that works from the cellular or even organismic whole. It may also be seen as the primary holder of the organized information spoken about in the previous chapter.

\subsection{Special Character of Interfacial Water}

The coherent cellular electromagnetic field and the polar oscillations are not the only issue that brings order into life. As life on the Earth is so strongly connected with water the latter should be examined from the standpoint of long range long term ordered states, too. The most important is the interfacial water facing hydrophilic surfaces. Regarding ordinary liquid water there are two basic outlooks: 1) according to the conventional and strongly established one water is normally in a state of chaotic movement, resulting in Brownian movement; 2) another (unconventional, but with many different indications and theoretical elaborations) sees water partially in a state of chaotic movement and partially ordered (depending on temperature and some other conditions, like the concentration of polar molecules or ions). The order should stem from phase transitions of water from the vapor phase to a condensed phase where all molecules oscillate in unison, in tune with a self-trapped electromagnetic field within extended mesoscopic space regions called coherent domains (CDs) (see [3] [12] [13] [14]). This basic water order was extensively researched by Preparata and Del Giudice. As many authors argue, coherence stabilizes these domains and makes them resistant to thermal chaos (noise).

There are further strong indications that water is even more complexly organized if facing hydrophilic surfaces (see the extensive work by Pollack and coworkers [15] [16]). It has many signs of long range long term order that could have static as well as dynamic characteristics. Since it tends to exclude solutes it 
was called "exclusion zone water" or simply EZ water. In organisms and various organic vesicles the relative amount of interfacial water is very high. Therefore, even if we take into account only the ordered state of water, we may assume a much higher order from the conventionally expected one within various quasi cellular structures (vesicles) that were quite plausible candidates for the emergence of the first organisms (like in Oparin's coacervates or Fox's microspheres).

The coherent oscillations regime in water solutions enables the active information to be widely dispersed and stable to a high measure. Coupling of ordered water regime (EZ or/and $\mathrm{CDs}$ ) to a rich and appropriate molecular system would in principle enable its evolution. The prebiotic system should be stable enough to ensure the continuation of the already established information complexity. This should be connected to the appearance of extended (i.e. higher hierarchical) coherent domains, strongly coupled to countless molecular interactions as described by Del Giudice [17]. On the other hand, the ordered systems (vesicles) should have been sufficiently mutable to enable their further evolution, since otherwise their development could got stuck and no true life would follow. In the following we shall examine empirically discovered systems, called nanobacteria that could demonstrate a highly ordered water regime coupled to a rich and appropriate molecular content.

\section{The Origin of Organisms from Organized Nano-Vesicles or Nanoparticles (NVs)}

\subsection{Living or Non-Living Entities?}

In the deficiency of any universal definition of life and for the purpose of this article we may define life as a highly organized, complex and dynamic process that takes place in matter using energy and information. It is capable of autonomous continuation and potentially limitless evolution by means of (differential) reproduction of its basic units (organisms).

According to this definition, prions-highly infectious agents causing mad cow disease (Bovine spongiform encephalopathy-BSE) are not living being since they apparently lack any possibility of limitless evolution. Viruses on the other hand are living beings, but only conditionally, as parasites, which mean that their life can take place only in other living beings. Only there their dynamically organized information can be expressed in matter (molecules).

Further, we have various vesicles that have been studied as possible stages in the origin of life process. Take for instance Oparin's coacervates [18] and Fox's microspheres [19]. In suitable environments they may demonstrate many characteristics of unicellular organisms, like growth, some form of metabolism, aggregation, propagation and strikingly, even some form of resting electric potential as well as excitability (action potential) [20].

From the standpoint of molecular and genocentric outlook of life these complex vesicles would never be treated as anything similar to organisms, not even as a proper path to them, maybe only as a suitable environment for a start of a true molecular origin of life process. The reason is that it is difficult to envision, 
how they could acquire the genetic apparatus with a functioning genetic code. But if we assume a different perspective that sees life in terms of organized information expressed through well ordered physical and physicochemical states, these vesicles become much more interesting and worthy of further and closer study.

\subsection{Nanobacteria and Their Biological Status}

In contemporary science, nanobacteria or more universally, nanoparticles ${ }^{2}, \mathrm{NVs}$, seem perfect forms to challenge our views about life and pre-biotic systems. Nanobacteria are formations that are some 5 - 100 times smaller from ordinary bacteria $(\sim 1 \mu)$ and were first found even with some genetic elements, although this was not universally confirmed. They were first identified in Italian hot springs in 1993 [23] resembling among other things carbonate microstructures found in the Allan Hills meteorite remnants from Mars that were (in 1996) hypothesized to represent Martian primitive life forms [24]. They were also found to accompany human life since they were discovered in all our bodily fluids and connected to many of our diseases, mostly to ones involving calcification (kidney stones, arteriosclerosis, tooth scale etc.).

While they were soon regarded as too small to be able to function as fully living beings by the majority of microbiologists, nanobacteria nevertheless demonstrated capability to contaminate cell cultures and even more, they proved to be resistant to ordinary sterilizing procedures like heat (for instance they may endure the heating for 1 hour at $90^{\circ} \mathrm{C}$ ) and many antibiotics [22] [23] [25]. They can form colonies larger than $1 \mathrm{~mm}$ and akin to coacervates and microspheres may exhibit budding and may propagate, even if much much slower than Escherichia coli at optimal conditions [25]. Their most usual property is that they appear as reproducing calcifying macromolecular vesicles [21] with something similar to cellular wall and filamentous surface projections.

Taken all these facts together, the behavior of these tiny bodies strongly resembles living organisms (they are highly biomimetic, at least). Their complex multiplication indicates that they posses organized information, discussed in chapter 2.1. And since we may be fairly convinced-at least for the great majority of them-that they do not posses the genetic apparatus distinctive for ordinary living beings and even viruses, their still very complex organization together with variable chemistry indicates a highly organized electrodynamic field discussed in chapter 2.2. But are they alive? The group around Martel and Young [22] [23] [26] claims that nanobacteria are only complex inorganic (mostly calcium carbonate or phosphate based) - organic (mostly protein) systems. These researchers managed to form nanobacteria like vesicles, called bions, on the basis of other cations than $\mathrm{Ca}$, like $\mathrm{Mn}, \mathrm{Cu}, \mathrm{Fe}, \mathrm{Ni}, \mathrm{Sr}, \mathrm{Na}$ etc. (see [26]), but still with animal proteins, e.g. fetal bovine serum. Bions also form aggregates and colonies and are mostly (but not all of them) round shaped and of various sizes. Being

${ }^{2}$ In [21] Kajander defines nanobacteria within the human body as calcifying nanoparticles. Martel et al. [22] extended this view since they treat nanobacteria as mineralo-organic nanoparticles. 
produced absolutely artificially, they have no trace of genetic material and are classified as non-living, bio-mimetic complex chemical systems [26]. Biomimetic forms may be achieved also by solely inorganic chemicals, like silica, barium and calcium and are called biomorphs; they were aptly described by the group around Garcia-Ruiz [27]. Both scientific teams warn before various attempts to identify extraterrestrial bacterial like microfossil forms (like the already mentioned one from Allan Hills, see also [24]) or paleontological sources as the signs of primitive living beings. Resemblance to living beings may rest only on biomimetic properties of such inorganic or organic-inorganic precipitations.

On the other hand, stemming from various researches finding that nanobacteria are susceptible to a number of chemicals there are authors claiming that nanobacteria are definitely living creatures and criticize the previously mentioned estimation (see [21] [25]). A further confirmation for their position they see in the nanobacteria needs for oxygen for their successful propagation. In their view nanobacteria are mostly infectious and harmful agents, usually where mineralization of tissues or teeth is at stake, but nevertheless also as only opportunistic companions of our life, since they were found also in healthy humans. Moreover, they also see possibility to heal some illnesses via appropriate chemicals together with nanobacteria [25] [28].

Therefore, we have two different outlooks on nanobacteria and NVs in general, both with their own proper argumentation. Which view is closer to truth? I will propose a bold hypothesis that both of them are right in their own way, representing two facets of the same reality. First, the dispute about the biological status of nanobacteria and NVs in general demonstrates the already mentioned embarrassing situation within biology that the latter has no general definition of its basic research theme-life. What from a certain standpoint may seem to be a living organism may from another view be regarded as a non-living system. Of course, this does not come to the fore when we think about indisputably living organisms like humans, animals and plants. But if we approach certain limits (usually connected with smallness), when some systems may still demonstrate certain characteristics of life, while lacking others, the absence of the life's definition presents a big challenge.

From the standpoint of the origin of life problem, we may raise a hypothesis that a broad span of NVs represents just a passage from the inanimate to the living world. Various synthetic bions and biomorphs may truly represent only very complex forms of precipitation (crystallization) of various chemicals. On the other hand, at least some nanobacteria-perhaps the ones found in bodily fluids - may represent factual living beings with the capability of adaptation and evolution. Somewhere in this strange nano-world there may run the demarcation line between life and non-life-and again, its position depends on our definition of life. In the light of this consideration, three important consequences logically follow:

a) The origin of life was not a moment in some distant geological past-it is all the time here with us, not as a "miraculous" moment, but as a permanent 
passage of certain (nano)vesicular systems (we may assume this is taking place within the realm of nanobacteria, but may also in bions).

b) The "origin of life" (more appropriately the emergence of living systems) is not so unfavorable, so an improbable event as it is imagined in the conventional biology.

c) This simplicity of the emergence of living beings strongly supports the assumption that life is not only a molecular phenomenon, but is primarily based on self-organizational capacities of mesoscopic (vesicular) systems that include not only their (robust) chemistry, but overall their physics and physical chemistry.

If we adopt the here offered definition of life that is not bound to its chemical basis, but to a specifically organized information, we may safely assume that our known and unambiguous (DNA-RNA-proteins) life (life 2) is surrounded and even interspersed by a parallel life (life 1) that is much more robust. Its robustness is proved by its already described high resistance against many for ordinary organisms highly detrimental chemical and physical conditions (even to a high gamma radiation, see for instance [25]). This high robustness indicates that life 1 is not organized on a detailed molecular informational matrix as it is the case with life 2, but is rather much more slack, only roughly defined in its chemistry. Yet, its forms are still relatively highly organized owing to their specific physical characteristics spoken about in the second chapter.

Even if we accept that at least some nanobacteria are living beings (although probably without DNA or RNA), the question still remains, whether these forms may transit to life 2 . This would warrant intensive further research. On the other hand it is already clear that life 2 may not only pass to life 1 , but it actually produces some of its forms. Life 1 can perhaps also be formed by an appropriate mixture and conditions of various inorganic and organic substances as the research work of bions by Reich showed (see [22] [29], the name "bions" used in the works by Martel and Young was taken just from Reich [22]). Reich speaks about moving creatures, got from purely sterilized conditions after autoclaving some living or formerly living material. They resembled paramecia or amoebas. These findings were confirmed also by long and systematic research by DeMeo [30]. In accord with our definition of life, if such systems are capable of further evolution in the sense of gradual organizing biochemistry, therefore having no handicap they can be classified as systems spanning between life and the inanimate, even if highly self organized, chemical systems.

\section{Conclusions}

Throughout the article, we tried to demonstrate that living organization does not exist only on the chemical level of complex molecular interconnected webs, but also, and maybe primarily, on specific physical and physicochemical characteristics of living matter with the long range order at its basis. It is still matter composed of atoms and molecules, but their exact composition is not so important in detail as it would hold for a system based solely on chemistry. The beauty of 
this outlook is that the long range regime in various vesicles or NVs in general can be highly organized even when the pre-biotic chemistry is in its infant stage and, when leaved to it, would prove to be incapable of any growth in informational (organizational) richness. Namely, from the empirical and theoretical study of autocatalytic chemical systems it is well known that even when such simple autocatalytic cycles can have some evolution (change of structure), sooner or later they would come to an unsurpassable edge, because of a too high imprecision in multiplication (transfer of their information from the parental to offspring molecules; see a brilliant study of Manfred Eigen [31] [32]). According to Eigen's research, strictly molecular evolution needs sufficient preciseness in replicating the genetic information carriers. However, NVs can replicate and reproduce themselves, but are at the same time chemically robust, i.e. at least majority of them have no exact molecular-genetic reproduction system. Following Eigen, we could conclude that their information content is too complex to enable them to reproduce solely on a molecular basis. Yet, they do replicate stably, the apparently posses organized information discussed in 2.1 .

The stability of their organized information should rest at least in great part on non-chemical principles-on the already discussed principles of physical and physicochemical properties of interfacial water (see chapter 2.3), coherent domains and Bose condensation of polar molecules oscillations (see chapter 2.2). The passage from complex chemical systems to the simplest living forms, exhibited by nanobacteria and some other NVs, need neither DNA nor RNA, no semi conservatively replicating molecule. If we consider life from this standpoint, it becomes understandable that under suitable conditions it may emerge from the inanimate nature much simpler than if it were based solely on chemistry. Contrary to first hand expectations, these systems are not rare or past, but abound here and now, around and even within us-they are mostly called nanobacteria. They were found in nature, in human fluids and may also be artificially synthesized. They may be combined inorganic-organic or only inorganic. Other names for them are nanobes, nanoparticles, bions and biomorphs, shortly nanovesicles, NVs. They are of varying sizes, may have complex morphologies and show capabilities of reproduction, a sort of metabolism and even parasitism. In some cases it was reported (though it should still be verified by more laboratories) that they may exhibit complex, beyond Brownian movement behavior (all these NVs when freely suspended in water demonstrate movement, since they fall within the range of Brownian regime).

In general, NVs may be regarded as a constantly existing passage from inanimate to fully animate systems (organisms). On one side of the special class of systems, we are faced with complexly crystallizing forms that may be treated as alive in the same measure as any ordinary crystals. On the other side, we find nanobacteria that-according to some authors and from the standpoint of more general definition of life-may be regarded as living creatures, even if they lack detailed molecular organization of ordinary present day life (life 2). They could be classified as life 1. According to many researches it is much more robust as 
life 2, which is also logical and it may be largely widespread on other planets as well, as the microfossils from Mars (Allan Hills meteorite remnants) clearly display.

Why then life 2 with its very fragile and complex biochemistry evolved from life 1 remains an open question and to be discussed in a later publication. In future, research of NVs should focus a) on the possibility of their evolution and adaptability, and b) the possibility already researched by Reich and DeMeo that they may exceed Brownian movement and thus demonstrate also kinetically that they are not only biomimetic but actually alive.

And if we come back to Pasteur and his famous proof that after 3 days his previously boiled soup remained clear, we may only comment that 3 days are said to be just an ordinary replication time for nanobacteria [25]. The nanobacteria were able only to double during his experiment. Perhaps he and the commission of the experiment were just too much quick to see that in due time the soup would actually change since there would abound strange life of nanobacteria. And maybe the history of biology since then would have been different.

\section{References}

[1] England J.L. (2013) Statistical Physics of Self-Replication. The Journal of Chemical Physics, 139, Article ID: 121923. https://doi.org/10.1063/1.4818538

[2] Jerman, I. (1998) Electromagnetic Origin of Life. Electro- and Magnetobiology, 17, 401-413. https://doi.org/10.3109/15368379809030740

[3] Jerman, I. (2016) The Origin of Life from Quantum Vacuum, Water and Polar Molecules. American Journal of Modern Physics. Special Issue: Academic Research for Multidisciplinary, 5, 34-43.

[4] Walker, S.I. and Davies, P.C.W. (2013) The Algorithmic Origins of Life. Journal of The Royal Society Interface, 10, Article ID: 20120869.

https://doi.org/10.1098/rsif.2012.0869

[5] Goodwin, B.C. (1984) A Relational or Field Theory of Reproduction and Its Evolutionary Implications, v: Beyond NeoDarwinism. Academic Press, London, 219-241.

[6] Goodwin, B.C. (1985) Developing Organisms as Self-Organising Fields, v: Mathematical Essays on Growth and the Emergence of Form. The University of Alberta Press, Edmonton, 185-200.

[7] Meijer, D.K.F. and Geesink, H.J.H. (2016) Phonon Guided Biology. NeuroQuantology, 14, 718-755. https://doi.org/10.14704/nq.2016.14.4.985

[8] Fröhlich, H. (1975) The Extraordinary Dielectric Properties of Biological Materials and the Action Of enzymes. Proceedings of the National Academy of Sciences, 72, 4211-4215. https://doi.org/10.1073/pnas.72.11.4211

[9] Fröhlich, H. (1988) Theoretical Physics and Biology. In: Frohlich, H., Ed., Biological Coherence and Response to Extemal Stimuli, Springer Verlag, Berlin, 1-24. https://doi.org/10.1007/978-3-642-73309-3 1

[10] Preparata, G. (1995) QED, Coherence in Matter. World Scientific, Singapore City. https://doi.org/10.1142/2738

[11] Fröhlich, H. (1978) Coherent Electric Vibrations in Biological Systems and the Cancer Problem. IEEE Transactions on Microwave Theory and Techniques, 26, 613-618. https://doi.org/10.1109/TMTT.1978.1129446 
[12] Del Giudice, E., Preparata, G. and Vitiello, G. (1988) Water as a Free Electric Dipole Laser. Physical Review Letters, 61, 1085-1088. https://doi.org/10.1103/PhysRevLett.61.1085

[13] Del Giudice, E., et al. (2005) Coherent Quantum Electrodynamics in Living Matter. Electromagnetic Biology \& Medicine, 24, 199-210. https://doi.org/10.1080/15368370500379574

[14] Bono, et al. (2012) Emergence of the Coherent Structure of Liquid Water, Water, 4, 510-532. https://doi.org/10.3390/w4030510

[15] De Ninno, A. (2016) Dynamics of Formation of the Exclusion Zone near Hydrophilic Surfaces. Chemical Physics Letters, 667, 322-326. https://doi.org/10.1016/j.cplett.2016.11.015

[16] Pollack, G.H. (2013) The Fourth Phase of Water. Ebner and Sons Publishers, Seattle.

[17] Giudice Del, E., Spinetti, P.R. Tedeschi, A. (2010) Water Dynamics at the Root of Metamorphosis in Living Organisms. Water, 2, 566-586.

https://doi.org/10.3390/w2030566

[18] Oparin, A.I. and Gladilin, K.L. (1980) Evolution of Self-Assembly of Probionts. BioSystems, 12, 133-145. https://doi.org/10.1016/0303-2647(80)90011-8

[19] Fox, S.W. and Nakashima, T. (1980) The Assembly and Properties of Protobiological Structures, BioSystems, 12, 155-166. https://doi.org/10.1016/0303-2647(80)90013-1

[20] Ishima, Y., et al. (1981) Electrical Membrane Phenomena in Spherules from Proteinoids and Lecithin. Biosystems, 13, 243-251. https://doi.org/10.1016/0303-2647(81)90004-6

[21] Kajander, E. (2006) Nanobacteria-Propagating Calcifying Nanoparticles. Letters in Applied Microbiology, 42, 549-552. https://doi.org/10.1111/j.1472-765x.2006.01945.x

[22] Martel, et al. (2014) Of Nanobacteria, Nanoparticles, Biofilms and Their Role in Health and Disease: Facts, Fancy and Future. Nanomedicine, 9, 483-499. https://doi.org/10.2217/nnm.13.221

[23] Young, J.D. and Martel, J. (2010) The Rise and Fall of Nanobacteria. Scientific American, 302, 52-59. https://doi.org/10.1038/scientificamerican0110-52

[24] Martel, J., et al. (2012) Biomimetic Properties of Minerals and the Search for Life in the Martian Meteorite ALH84001. Annual Review of Earth and Planetary Sciences, 40, 167-193. https://doi.org/10.1146/annurev-earth-042711-105401

[25] Yaghobee, S., et al. (2015) What Are the Nanobacteria? Biotechnology \& Biotechnological Equipment, 29, 826-833. https://doi.org/10.1080/13102818.2015.1052761

[26] Wu, C.-Y., et al. (2013) Bions: A Family of Biomimetic Mineralo-Organic Complexes Derived from Biological Fluids. PloS ONE, 8, e75501.

[27] García-Ruiz, J.M., et al. (2009) Morphogenesis of Self-Assembled Nanocrystalline Materials of Barium Carbonate and Silica. Science, 323, 362-365. https://doi.org/10.1126/science.1165349

[28] Lin, Y., Zheng, R., He, H., et al. (2009) Application of Biomimetic Mineralization: A Prophylactic Therapy for Cracked Teeth? Medical Hypotheses, 73, 493-494. https://doi.org/10.1016/j.mehy.2009.05.050

[29] Reich, W. (1938) The Bion Experiments on the Origin of Life.

[30] DeMeo, J. http://www.orgonelab.org/DeMeoBionsColor.pdf

[31] Eigen and Schuster (1979) The Hypercycle: A Principle of Natural Self-Organization. 
Springer Verlag, New York.

[32] Eigen, M., et al. (1981) The Origin of Genetic Information. Scientific American, 244, 88-118. https://doi.org/10.1038/scientificamerican0481-88

\section{Abbreviation List}

BSE-bovine spongiform encephalopathy ( $\operatorname{mad}$ cow disease), disease based on a deformed prion molecule within the brain of mammals.

$\mathrm{CD}$-coherent domain (CDs plural). A cohenrently vibrating area of water (or any liquid) that is not static, but very dynamic.

DNA-deoxyribonucleic acid, the essential molecular carrier of genetic information.

EZ water-(exclusion zone water) a few hundreds of microns thick layer of water bordering to hydrophilic surface. It excludes most solutes and is consequently clear.

$\mathrm{Mn}, \mathrm{Cu}, \mathrm{Fe}, \mathrm{Ni}, \mathrm{Sr}, \mathrm{Na}, \mathrm{Ca}$-various cations (metal ions), see Periodic system. $\mathrm{NVs}$-nanoparticles or nanovesicles, composite particles in water spanning from few nanometers up to or even exceeding a micron.

RNA-ribonucleic acid, a macromolecule involved in genetic mechanisms, may be a carrier of information (RNA viruses) or the messenger of it (mRNA) or be involved in genetics in other ways (tRNA, rRNA).

Submit or recommend next manuscript to OALib Journal and we will provide best service for you:

- Publication frequency: Monthly

- 9 subject areas of science, technology and medicine

- Fair and rigorous peer-review system

- Fast publication process

- Article promotion in various social networking sites (LinkedIn, Facebook, Twitter, etc.)

- Maximum dissemination of your research work

Submit Your Paper Online: Click Here to Submit

Or Contact service@oalib.com 